\title{
Effect of SPIO Nanoparticle Concentrations on Temperature Changes for Hyperthermia via MRI
}

\author{
Alsayed A. M. Elsherbini ${ }^{1}$ and Ahmed El-Shahawy ${ }^{2}$ \\ ${ }^{1}$ Photochemistry and Nanochemistry, National Institute of Laser Enhanced Science (NILES), Cairo University, \\ P.O. Box 12613, Giza, Egypt \\ ${ }^{2}$ Children's Cancer Hospital, Cairo 57357, Egypt
}

Correspondence should be addressed to Alsayed A. M. Elsherbini; elsayed@niles.edu.eg

Received 25 November 2012; Accepted 23 February 2013

Academic Editor: Mohamed Bououdina

Copyright (C) 2013 A. A. M. Elsherbini and A. El-Shahawy. This is an open access article distributed under the Creative Commons Attribution License, which permits unrestricted use, distribution, and reproduction in any medium, provided the original work is properly cited.

\begin{abstract}
Magnetic nanoparticles (MNPs) are being developed for a wide range of biomedical applications. In particular, hyperthermia involves heating the MNPs through exposure to an alternating magnetic field (AMF). These materials offer the potential for selectively by heating cancer tissue locally and at the cellular level. This may be a successful method if there are enough particles in a tumor possessing sufficiently high specific absorption rate (SAR) to deposit heat quickly while minimizing thermal damage to surrounding tissue. The current research aim is to study the influence of super paramagnetic iron oxides $\mathrm{Fe}_{3} \mathrm{O}_{4}(\mathrm{SPIO})$ NPs concentration on the total heat energy dose and the rate of temperature change in AMF to induce hyperthermia in Ehrlich carcinoma cells implanted in female mice. The results demonstrated a linearly increasing trend between these two factors.
\end{abstract}

\section{Introduction}

Hyperthermia offers an attractive approach for the treatment of cancer as a local therapy. It is associated with fewer side effects in comparison to chemotherapy and radiotherapy, and it can be used in combination with all conventional treatment modalities. But hyperthermia has not yet been established in clinical routine; this is mostly not due to a general lack of efficacy but rather to the limitations of the currently available techniques with respect to selectively targeting the tumor region and homogenously distributing the heat within the tumor [1]

Magnetic induced interstitial hyperthermia addresses these shortcomings, especially for deep-seated and poorly accessible tumors. For this application, MNPs exhibiting super paramagnetic behavior at room temperature are preferred because they do not retain any magnetism after removal of the magnetic field. Furthermore, the particles must have combined properties of high magnetic saturation, biocompatibility, and interactive functions at the surfaces. Among them, iron oxide particles such as magnetite $\left(\mathrm{Fe}_{3} \mathrm{O}_{4}\right)$ or its oxidized form maghemite $\left(\gamma-\mathrm{Fe}_{2} \mathrm{O}_{3}\right)$ are by far the most commonly employed in biomedical applications, since their biocompatibility has already been proven by Schwertmann, 1991 [2]. Highly magnetic materials such as cobalt and nickel are toxic, susceptible to oxidation and hence are of little interest.

Ideally, in hyperthermia treatment, one would optimize the temperature pattern. However, temperature profiles in biological tissues during hyperthermia depend on the combined influences of applied power and several cooling mechanisms. These mechanisms are difficult to predict, since cooling depends heavily on thermal parameters that vary in time. Therefore, hyperthermia treatment limits to SAR predictions and assumed that a high SAR level will correlate with a high temperature [3].

Previous studies showed that the heating capacity of MNPs can be influenced by many factors; for example, the heating effect of magnetic particles depended strongly on the particles size [4]. The magnetic anisotropy constant can influence the heating mechanism of MNPs $[5,6]$. The strength of the applied AMF [7]. On the other hand Portet et al. 
demonstrated that the type of surfactant coats the MNPs has influence on the heating capacity of MNPs [8]. The influence of MNPs concentration on the heat energy and hence the temperature change was the interest in this study.

\section{Materials and Methods}

2.1. Materials. All chemicals used in this study were analytical grade reagents and were used without further purification.

\subsection{Methods}

2.2.1. Preparation of SPIO NPs $\mathrm{Fe}_{3} \mathrm{O}_{4}$. A photochemical method was carried out by dissolving a mixture of ferrioxalate with $\mathrm{FeCl}_{3} \cdot 6 \mathrm{H}_{2} \mathrm{O}$ in predetermined concentration ratio in $100 \mathrm{~mL}$ of $10 \%$ aqueous hydrogen peroxide solution. The $\mathrm{pH}$ of the solution was adjusted to 13 by the addition of $1 \mathrm{~mL}$ of $\mathrm{NaOH}$. The black precipitates appeared just after the mixing of iron hydroxide with the base solution. These precipitates were collected by magnet, and the product was washed several times with distilled water and finally dried in the air. To increase the stability of SPIO NPs especially in vivo conditions, the prepared SPIO NPs were suspended in glycerin medium.

\subsubsection{Characterizations}

(1) Transmission Electron Microscopy (TEM). Transmission electron microscopy of the produced samples was formed by drying a drop of the solution on a carbon-coated copper grid. Particles sizes were determined from the micrographs of the Joel-100S transmission electron microscope of resolution of $0.3 \mathrm{~nm}$.

(2) X-Ray Diffraction Analysis. Crystallographic study was performed on iron oxide powder by Rotating Anode Xray Diffractometer (Rigaku, Japan) using $\mathrm{Cu} \mathrm{Ka}$ radiation. The inter-planer distances $(d)$ were calculated according to Scherrer's equation and were compared to the JCPDS file $2.532 \mathrm{X}$-ray diffraction data in order to deduce the crystal structure.

2.2.3. Preparation of Tumor Bearing Mice. 6-8-week old female Swiss albino mice (20.0 to $29.2 \mathrm{~g}$, median, $26.3 \mathrm{~g}$ ) were injected with Ehrlich Carcinoma (EAC) from the breeding unit of the Egyptian National Cancer Institute (ENCI). $1 \times 10^{6}$ (EAC) cells/mL were implanted by subcutaneous inoculation to obtain a single grafted tumor. Ehrlich carcinoma cells were implanted in all mice at the same day and under the same conditions. During implantation mice were anesthetized by Fentanyl-dihydrochloride (Fentanyl; Janssen-Cilag, Neuss, Germany) $50 \mathrm{mg} / \mathrm{kg}$ body weight. The tumors started to appear within seven days after the day of implantation. Twenty days from the day of implantation, all tumors volumes for all mice were measured by digital caliper in three orthogonal diameters $\left(D_{1}, D_{2}\right.$, and $\left.D_{3}\right)$ :

$$
\text { Tumor Volume }=\left(D_{1} D_{2} D_{3}\right)\left(\frac{\pi}{6}\right) \text {. }
$$

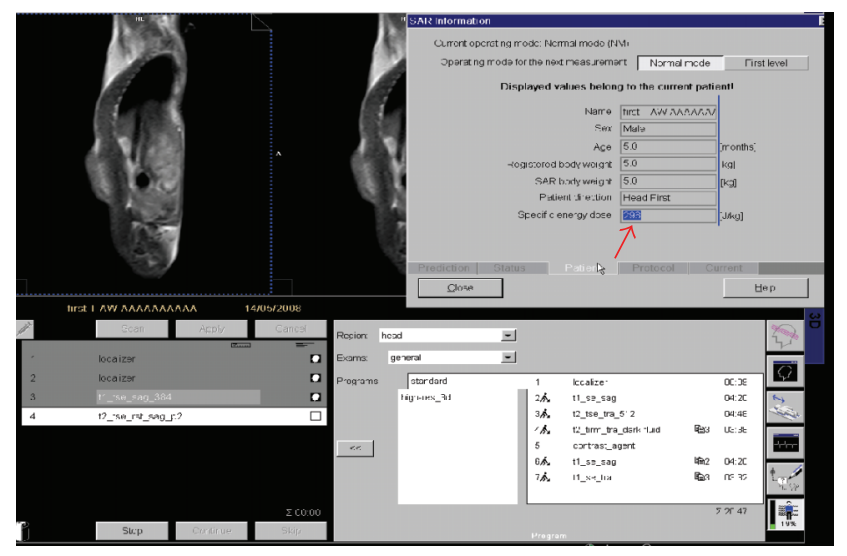

FIGURE 1: Typical photograph of Syngo software: the red arrow refer to SAR value by $\mathrm{J} / \mathrm{kg}$.

2.2.4. Experimental Design. After four weeks from the day of tumor implantation and when a grossly visible and palpable mass of the tumor was obtained, the experiments started. The tumors volumes measurements of the selected mice $(n=60)$ revealed no significant difference $P>0.3$ and the mean initial value of the tumors volumes was $72.5 \pm 3.2 \mathrm{~mm}^{3}$. The selected mice were classified into two groups as follow.

(i) The 1st group: the mice were not injected by SPIO NPs but subjected to AMF, that is, considered as baseline (control group $n=10$ ).

(ii) The 2nd group: the mice were subjected to AMF after direct injection by SPIO NPs with different concentrations into the region of interest (ROI). This group (SPIO + AMF) in turn was classified into (5 subgroups): the mice in the 1st subgroup $(n=$ 10) were injected by $1 \mathrm{~mL}$ from magnetic suspension containing $200 \mu \mathrm{g} \mathrm{Fe}_{3} \mathrm{O}_{4} / \mathrm{kg}$ body weight, the 2nd ( $n$ $=10)$ by $400 \mu \mathrm{g}$, the $3 \mathrm{rd}(n=10)$ by $600 \mu \mathrm{g}$, the 4 th $(n$ $=10)$ by $800 \mu \mathrm{g}$, and the 5 th $(n=10)$ by $103 \mu \mathrm{g}$.

Each mouse in these subgroups was subjected to AMF three times at 7, 14, and 21 days from the starting date of the experiment. All factors were fixed in all experiments (field strength, frequency, amplitude, size, and even shape of NPs). The values of total heat energy dose or in other wards specific energy dose (SED jg ${ }^{-1}$ ) for each concentration in the Ehrlich tumor were monitoring directly during the magnetic resonance imaging (MRI) via highly advanced soft ware (Syngo. NUMARIS/4, Siemens, German) (Figure 1) and consequently the heat deposition rate (HDR $\mathrm{jg}^{-1} \mathrm{~s}^{-1}$ ), maximum temperature achieved in the tumor and the time to maximum temperature (TMT). It is worth mentioning that the injected SPIO NPs were identified inside the tumor by electron microscopic image comparing with the control sample Figure 2. All experiments were approved by the regional animal care committee.

2.2.5. In Vivo Experiment. Four weeks after tumors implantations, mice were anesthetized with $2 \%$ to $3 \%$ isoflurane 


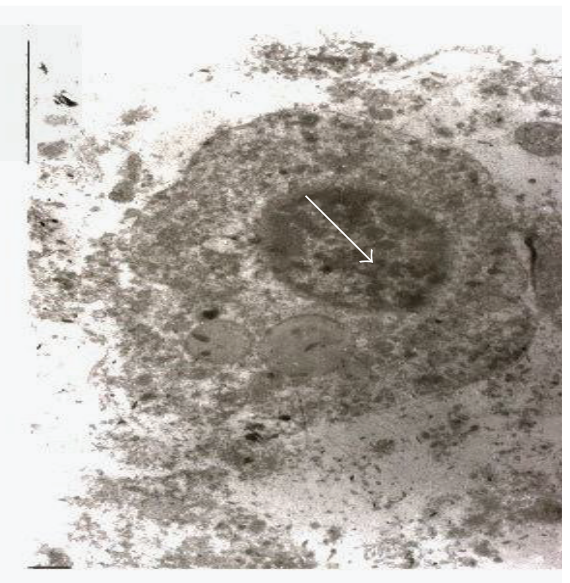

(a)

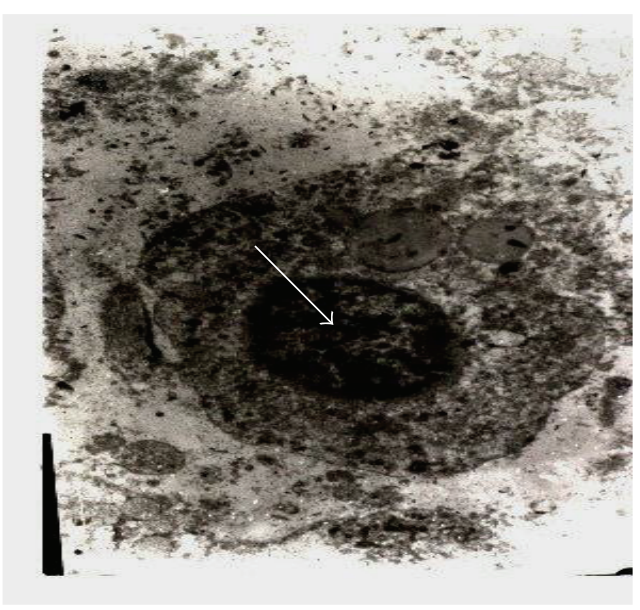

(b)

FIGURE 2: Transmission electron microscope image of (a) the control sample and (b) the injected SPIO NPs inside the tumor.

through an MR-compatible mobile inhalation system (DRE, Inc., Louisville, KY, USA). Three fiber optical temperature probes which are not RF sensitive (FISO, Inc., Quebec, Canada): the first probe was inserted in the center of the tumor, the second probe was placed immediately adjacent to the tumor periphery, and the third probe was inserted $1 \mathrm{~cm}$ inside the rectum. The optical fiber positions were monitored by radiography (Figure 3 ). After placing the probes, each mouse was centered inside the magnetic field for MRI. To focus the AMF on the region of interest, a surface transmit and receiver radiofrequency (RF) induction coil (inner diameter $7 \mathrm{~cm}$; length $7 \mathrm{~cm}$ ) specially designed for small animal imaging was placed on the center of tumor area in all experiments. All imaging studies were performed at the Children's Cancer Hospital $(\mathrm{CCH})$, department of radiology, using 1.5-Tesla high field MR scanner (Magnetom, Espree, Siemens, Germany) equipped with high-performance gradients $(23 \mathrm{mT} / \mathrm{m}$ maximum amplitude $120 \mathrm{~T}$ per $\mathrm{m}$ per sec maximum slew rate) and fast receiver hardware (bandwidth

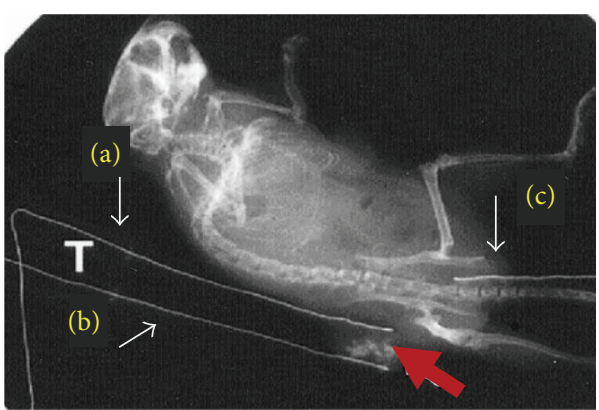

Figure 3: X-ray image (top view), T optical fiber thermocouples with measuring junctions located at tumor (a), tumor periphery (b), and at the rectum (c). The tumor was loaded with SPIO nanoparticles (red arrow).

$\pm 500 \mathrm{MHz}$ ). All imaging parameters were optimized for all experiments as follow.

Slice thickness $=2 \mathrm{~mm}$; interspaces $=1 \mathrm{~mm}$. Field of view $(\mathrm{FOV})=160 \mathrm{~mm}$, average $=15$, matrix $=256 \times 256$, slices $=12$ for an acquisition, power $=25 \mathrm{~kW}$, duty $=100 \%$, bandwidth $=9.62 \mathrm{kHz}$, and the variable factor was only the duration of exposure which was limited between 30 to 40 mins or until the rectal temperature of the mouse reached $41.5^{\circ} \mathrm{C}$ to prevent unnecessary mortality in the mice due to exposure to excessively high temperatures. All mice were subjected to AMF after 15 minutes from localized injection with SPIO NPs which were directly injected into the central portion of the tumors over a 5 min period; the slow injection rate was necessary because of the high interstitial tumor tissue pressure. The NPs were injected at more than one site to obtain a homogenous distribution. At the end of each experiment, mice were placed on their backs on a warm recovery bed. Each mouse was left until the core (rectal) temperature began to decrease and the probe was removed. When the righting reflex returned, mice were returned to their cages and observed for 48 hours for signs of morbidity. Dead mice were sent to necropsy for examination.

\section{Results}

\subsection{Characterization of MNPs}

3.1.1. Transmission Electron Micrograph (TEM). Transmission electron microscopy for the prepared SPIO NPs showed that particles have $\sim$ spherical shapes and their sizes were in the range of $\sim 54 \pm 3 \mathrm{~nm}$ (Figure 4 ).

3.1.2. X-Ray Diffraction Analysis. The XRD spectrum of the prepared SPIO NPs shows six characteristic peaks for $\mathrm{Fe}_{3} \mathrm{O}_{4}$ $\left(2 \theta=30.1^{\circ}, 35.5^{\circ}, 43.1^{\circ}, 53.4^{\circ}, 57.0^{\circ}\right.$, and $\left.62.6^{\circ}\right)$ marked by their indices $(220,311,400,422,511$, and 440$)$, respectively (Figure 5).

\subsubsection{Heating Capacity of MNPs}

(1) Monitoring SED and HDR. The mean values reported of the total heat energy dose were $\left[282.1 \pm 13.8 \mathrm{jg}^{-1}\right.$ for $(200 \mu \mathrm{g})$, 


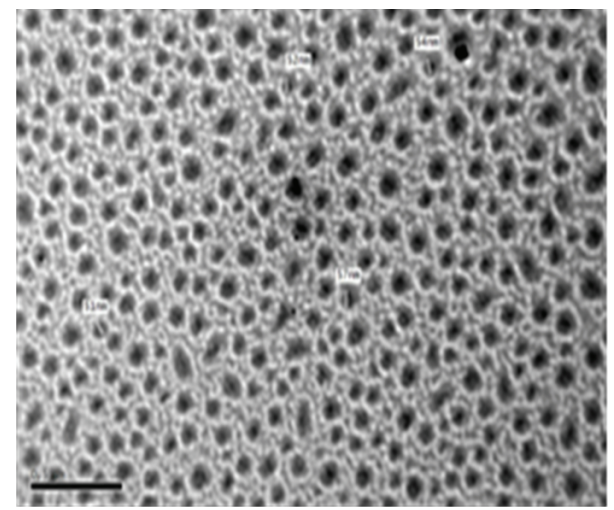

FIGURE 4: Transmission electron microscope of prepared SPIO NPs.

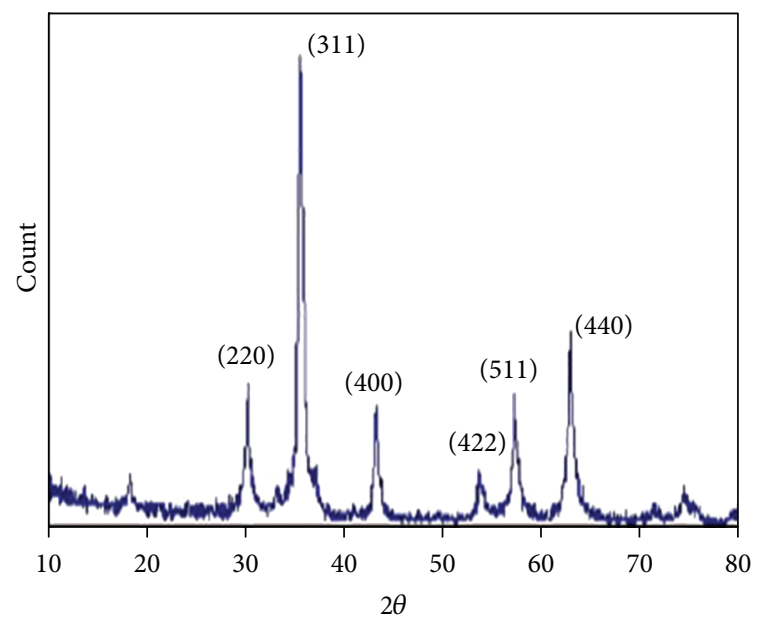

FIGURE 5: XRD patterns of prepared SPIO NPs.

$462.7 \pm 10.0$ for $(400 \mu \mathrm{g}), 663.7 \pm 13.0$ for $(600 \mu \mathrm{g}), 864.1 \pm$ 16.6 for $(800 \mu \mathrm{g})$, and $1087 \pm 18$ for $\left.\left(10^{3} \mu \mathrm{g}\right)\right]$.

The quantitative statistical analysis using Kruskal-Wallis test for comparison between more than two groups not only revealed that the SED values were directly proportional to the concentrations of the injected nanoparticles inside the tumors but also varied considerably between the different concentrations with highly significant $P$ values, at 7 days $P$ $<.001$, at 14 days $P<.001$, and at 21 days $P<.007$, but the variations in the SED values of each concentration between the $(7,14,21)$ days were insignificant and, $P<.7$ as shown in Figure 6.

The results of HDR revealed that the mean values of HDR in the tumors were [0.157 for $(200 \mu \mathrm{g}), 0.259$ for $(400 \mu \mathrm{g}), 0.367$ for $(600 \mu \mathrm{g}), 0.478$ for $(800 \mu \mathrm{g})$, and 0.604 for $\left.\left(10^{3} \mu \mathrm{g}\right)\right]$. These values varied considerably between the different concentrations of SPIO NPs with highly significant $P$ values, at 7 days $P<.001$, at 14 days $P<.001$, and at 21 days $P<.007$. Also, the variations in the HDR values of each concentration between the $(7,14$, and 21$)$ days were insignificant $P<.7$ (Figure 7).

(2) Monitoring Maximum Temperature and TMT. The maximum temperatures achieved inside the tumor were $[40.11 \pm$

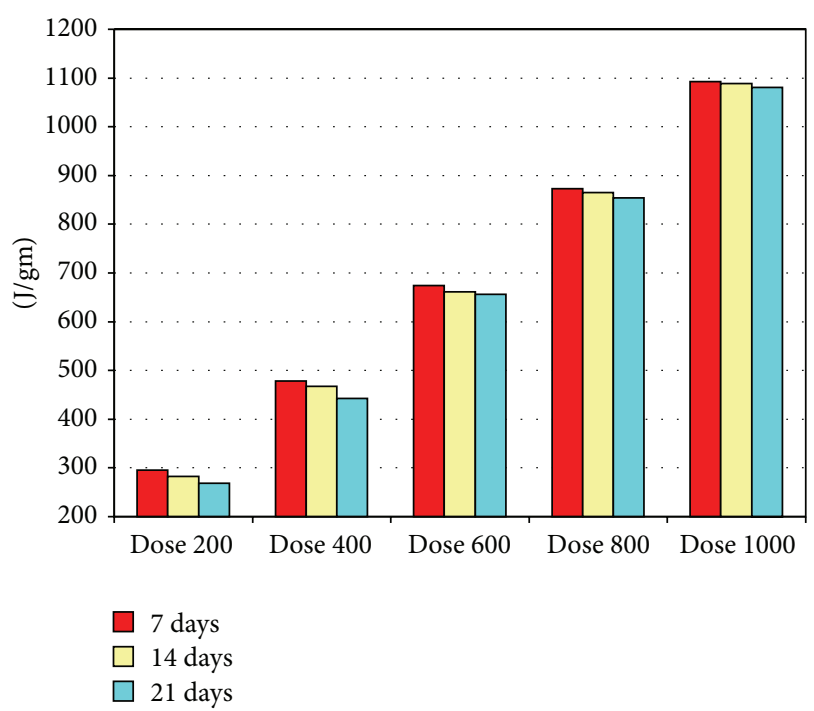

FIgURE 6: Shows Mean of SAR by jgm ${ }^{-1}$ dependent on SPIO NPs concentrations in three sessions (at 7,14 and 21 days from starting the experiments).

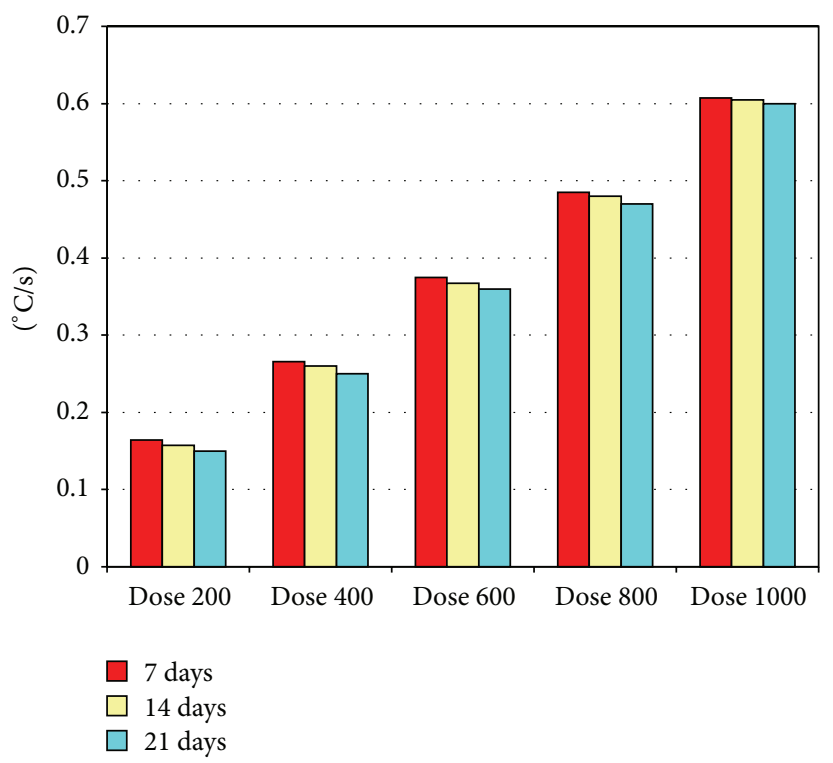

Figure 7: Mean values of HDR by $\left(\mathrm{jg}^{-1} \mathrm{~S}^{-1}\right)$ dependent on SPIO NPs concentrations in three sessions (at 7, 14, and 21 days from starting the experiments).

$1.52^{\circ} \mathrm{C}$ for $(200 \mu \mathrm{g}), 42.36 \pm 1.54^{\circ} \mathrm{C}$ for $(400 \mu \mathrm{g}), 44.43 \pm 2.0^{\circ} \mathrm{C}$ for $(600 \mu \mathrm{g}), 46.8 \pm 1.5^{\circ} \mathrm{C}$ for $(800 \mu \mathrm{g})$, and $48.6 \pm 1.0^{\circ} \mathrm{C}$ for $\left.\left(10^{3} \mu \mathrm{g}\right)\right]$ (Figure 8). The TMT recorded as $(40 \pm 2.5 \mathrm{~min}$ for 200 and $400 \mu \mathrm{g}, 30 \pm 2.0 \mathrm{~min}$ for $600 \mu \mathrm{g}, 25 \pm 5.0 \mathrm{~min}$ for $800 \mu \mathrm{g}$ and $20 \pm 5.0 \mathrm{~min}$ for $10^{3} \mu \mathrm{g}$ (Figure 9). It is worthwhile to mention that there was no substantial temperature elevation observed in the control group. 


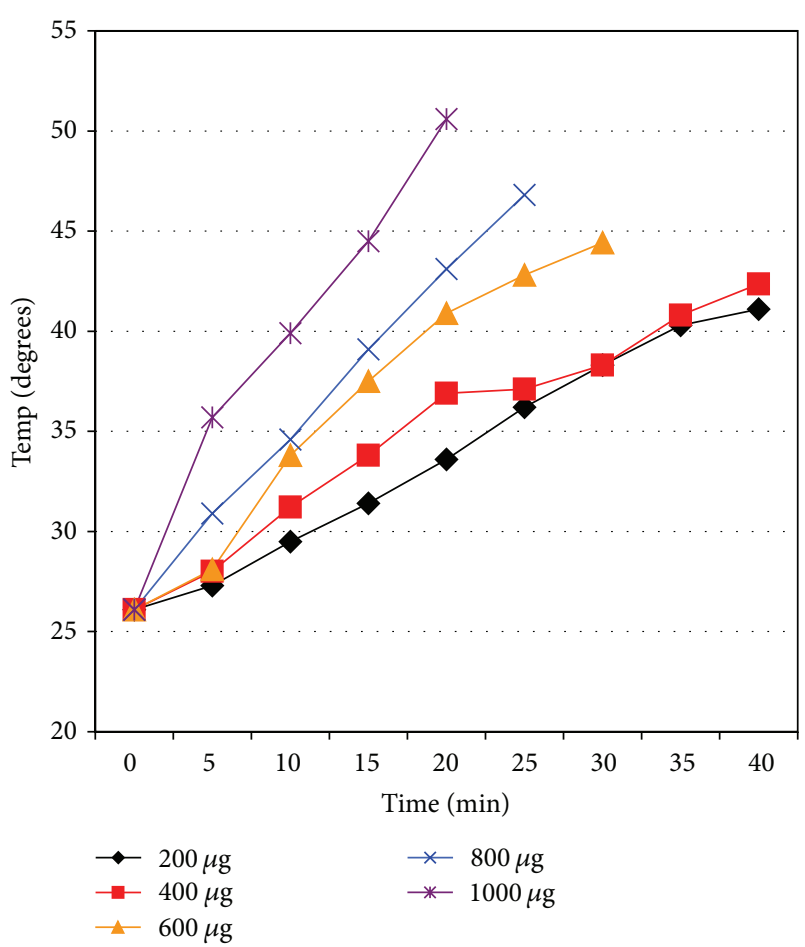

FIGURE 8: The maximum temperature achieved inside the Ehrlich tumors related with the concentrations of the injected SPIO NPs versus time. Data reported represents the mean values in the three sessions (7, 14, and 21 days from starting the experiments).

\section{Discussion}

In the current study, the SPIO NPs $\left(\mathrm{Fe}_{3} \mathrm{O}_{4}\right)$ were synthesized in solution under controlled conditions by wet chemical method. The size, morphology, uniformity, and crystal structure of the prepared SPIO NPs were determined using two techniques TEM and XRD which have been widely employed for this purpose. The TEM images revealed the quasispherical uncoated SPIO NPs with size changes in the range of $\sim 54 \pm$ $3 \mathrm{~nm}$. X-ray diffraction spectrum gave further information about the structure of the SPIO NPs. The positions and relative intensities of all diffraction peaks matched well with those from the JCPDS card (no. 85-1436) for $\mathrm{Fe}_{3} \mathrm{O}_{4}$ magnetite.

The heating ability of MNPs in the presence of AMF is usually expressed by the SAR, also called specific power loss (SPL) which can be defined as the amount of heat generated per unit gram per unit time. The reported SED value on Syngo represents the total heat dose and reflects a cumulative effect which differs according to the SAR of each MNPs concentration.

It is important therefore to understand the underlying physical mechanisms by which heat is generated. The heat generated by MNP via AMF may be due to three major mechanisms which depend greatly on the particle size and their magnetic properties. Large multidomain ferro- or ferrimagnetic materials contain several subdomains each having their own specific magnetization direction. When these materials are exposed to the AMF, the subdomain with magnetization

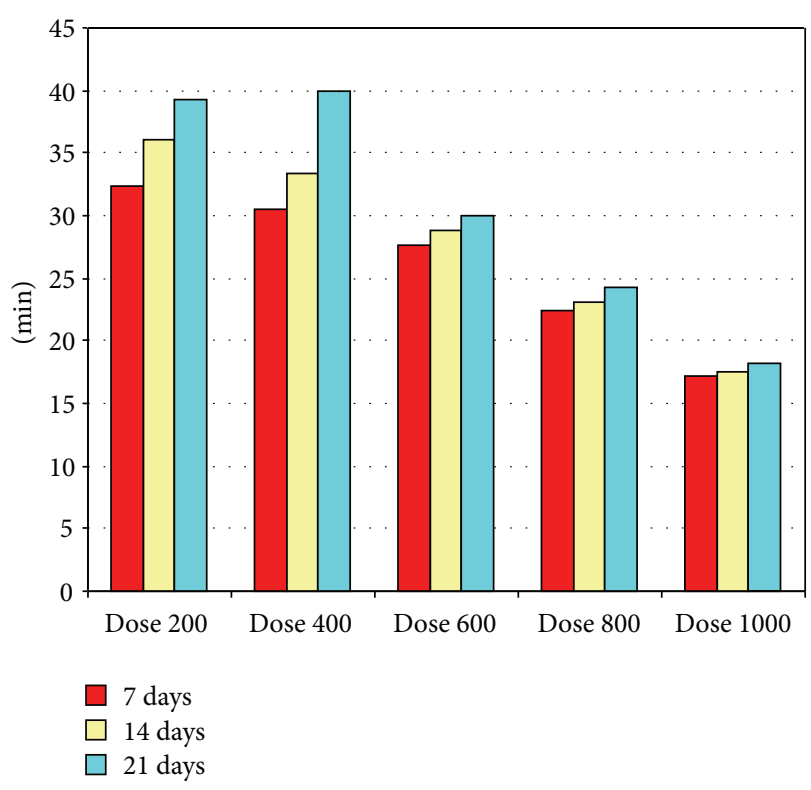

FIGURE 9: Mean values of TMT in the three sessions of treatment (7, 14 , and 21 days from starting the experiments).

direction along the magnetic field axis elongates and the other ones shrink. This leads to "domain wall displacements." Since the magnetization curves for increasing and decreasing magnetic field amplitudes do not coincide, the material demonstrates "hysteresis behavior" and produces heat. In small particles such as SPIO NPs, there is no domain wall, and therefore, hysteresis losses cannot occur. However, the external AMF energy helps magnetic moments to rotate and overcome the anisotropy energy barrier. This energy is then dissipated as heat when the particle relaxes to its equilibrium orientation. This mechanism is called Néel relaxation. There is also a third mechanism called Brownian relaxation that causes both multidomain and single domain particles to heat up. In this process, energy barrier for reorientation of a particle is given by rotational friction due to the rotation of the entire magnetic particle caused by the AMF torque force on the magnetic moment of the particle [9].

The most effective mechanism of heat generation in the current study may be related to Brownian relaxation; this is due to that the SPIO NPs were suspended in glycerin which is a highly viscous medium which in turn increases the friction loss where the source for heat generation relies in this case on the friction processes between the NPs and the medium during the physical rotation of the particle induced by the alternation of the external magnetic field.

There are several factors affect on the SAR values. Robert et al. 2005 [10] demonstrated that the heating potential of MNPs for inducing hyperthermia depends on the saturation magnetization, magnetic field strength, field amplitude, duty, duration, type of NPs, size, size distribution, particle structure, shape, and interparticle interactions, all affect the SAR values of the SPIO NPs. However, each contributes in different ways and with different magnitudes and may even be in competition with each other. So, it is very difficult to 
give a general theoretical expression of SAR because several equations may be proposed, but these theoretical trends are not always validated by experimental SAR measurements.

Adding to the above factors, the concentration effect was studied in the current research. The results revealed that increasing the concentrations of injected MNPs resulted in increasing the SAR and hence the higher SED and HDR values; all of these lead to increasing the maximum temperature achieved and decreasing the TMT (Figures 69). It is worthwhile to mention that the total heat dose values acquired in this study were lower than those of other studies, Dennis et al., 2008 [11], but the time required to reach the therapeutic temperature was short compared to the previous studies, Giri et al., 2005 [12].

It is well known that for hyperthermia treatment enough heat must be generated by the particles to sustain tissue temperature of at least $42^{\circ} \mathrm{C}$ for 30 minutes. Indeed this condition was achieved in the current study by using different concentrations $\left(400-10^{3} \mu \mathrm{g} / \mathrm{kg}\right.$ ). The optimum concentration of the magnetic suspension was $800 \mu \mathrm{g} / \mathrm{kg}$, where the amount of total head dose was sufficient to increase the temperature above $42^{\circ} \mathrm{C} \sim 45^{\circ} \mathrm{C}$ in short time $\sim 20 \mathrm{~min}$ as shown in Figure 8 compared to $(400,600 \mu \mathrm{g})$ in the same figure. On the other hand, using high concentration $10^{3} \mu \mathrm{g}$ resulted in high SED values in short time; nevertheless the percentage of mortality was $80 \%$, and this may be attributed to high concentration resulting in high over stress on mice under AMF.

In this study, we used two physically different techniques, the magnetic resonance phenomena for acquiring imaging and treatment at the same time. By using noninvasive AMF for MR imaging through its interaction with the protons of the hydrogen atoms of the water molecules (MR images not shown), and at the same time inducing thermal effect on cancer cells through induction of magnetic gradients generated by a clinical MRI system. Although the two techniques occur at the same time, they are completely different in the physical theory and mechanism.

\section{Conclusion}

The current preliminary study demonstrated that the temperature changes inside tumors related with the variations of SED and HDR values which in turn depend directly on the injected concentrations of magnetic suspension inside tumors.

\section{References}

[1] P. Wust, B. Hildebrandt, G. Sreenivasa et al., "Hyperthermia in combined treatment of cancer," Lancet Oncology, vol. 3, no. 8, pp. 487-497, 2002.

[2] X. Liang, K. Exu, and X. Jianhe, "Preparation of Immunomagnetic nanoparticles and their application in the separation of mouse CD34 ${ }^{+}$hematopoietic stem cells," Journal of Magnetism and Magnetic Materials, vol. 321, pp. 1885-1888, 2009.

[3] B. Thiesen and A. Jordan, "Clinical applications of magnetic nanoparticles for hyperthermia," International Journal of Hyperthermia, vol. 24, no. 6, pp. 467-474, 2008.
[4] M. H. Khedr, A. A. Omar, and S. A. Abdel-Moaty, "Magnetic nanocomposites: preparation and characterization of Co-ferrite nanoparticles," Colloids and Surfaces A: Physicochemical and Engineering Aspects, vol. 281, no. 1-3, pp. 8-14, 2006.

[5] J. P. Fortin, C. Wilhelm, J. Servais, C. Ménager, J. C. Bacri, and F. Gazeau, "Size-sorted anionic iron oxide nanomagnets as colloidal mediators for magnetic hyperthermia," Journal of the American Chemical Society, vol. 129, no. 9, pp. 2628-2635, 2007.

[6] D. L. Zhao, X. W. Zeng, Q. S. Xia, and J. T. Tang, "Preparation and coercivity and saturation magnetization dependence of inductive heating property of $\mathrm{Fe}_{3} \mathrm{O}_{4}$ nanoparticles in an alternating current magnetic field for localized hyperthermia," Journal of Alloys and Compounds, vol. 469, no. 1-2, pp. 215-218, 2009.

[7] A. Jordan, P. Wust, H. Faehling, and J. Krause, "Inductive heating of ferrimagnetic particles and magnetic fluids: physical evaluation of their potential for hyperthermia," International Journal of Hyperthermia, vol. 9, pp. 51-57, 1993.

[8] D. Portet, B. Denizot, E. Rump, J. J. Lejeune, and P. Jallet, "Nonpolymeric coatings of iron oxide colloids for biological use as magnetic resonance imaging contrast agents," Journal of Colloid and Interface Science, vol. 238, no. 1, pp. 37-42, 2001.

[9] M. Ma, Y. Wu, J. Zhou, Y. Sun, Y. Zhang, and N. Gu, "Size dependence of specific power absorption of $\mathrm{Fe}_{3} \mathrm{O}_{4}$ particles in AC magnetic field," Journal of Magnetism and Magnetic Materials, vol. 268, no. 1-2, pp. 33-39, 2004.

[10] I. Robert, J. Sally, D. Wolfgang, and C. Robert, "Application of high amplitude alternating magnetic fields for heat induction of nanoparticles localized in cancer," Clinical Cancer Research, vol. 1, no. 11, pp. 7093s-7103s, 2005.

[11] C. Dennis, A. Jackson, and J. Borchers, “"The influence of magnetic and physiological behavior on the effectiveness of iron oxide nanoparticles for hyperthermia," Journal of Physics D: Applied Physics, vol. 41, Article ID 134020, 2008.

[12] J. Giri, P. Pradhan, T. Sriharsha, and D. Bahadur, "Preparation and investigation of potentiality of different soft ferrites for hyperthermia applications," Journal of Applied Physics, vol. 97, no. 10, pp. 1-3, 2005. 

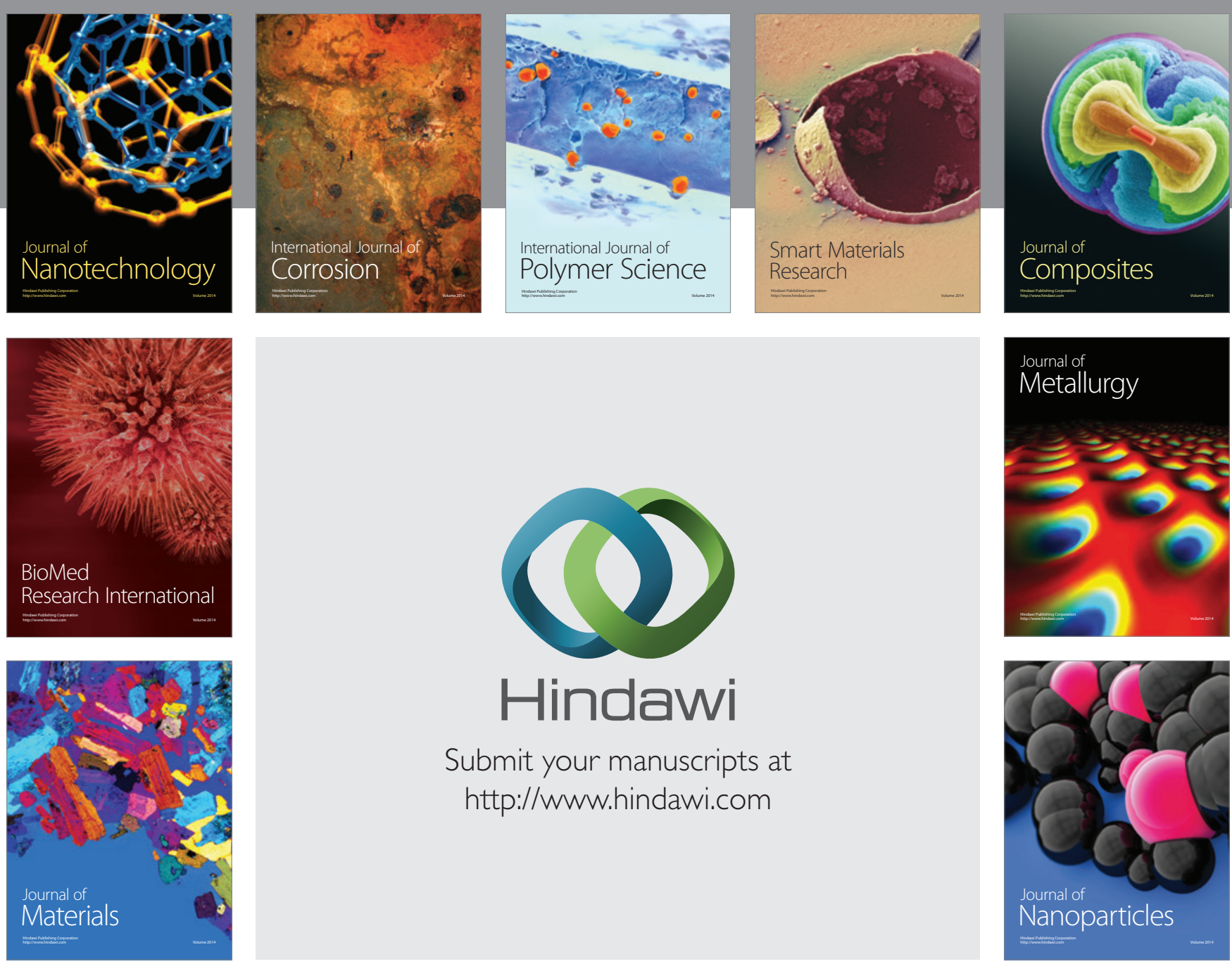

Submit your manuscripts at http://www.hindawi.com
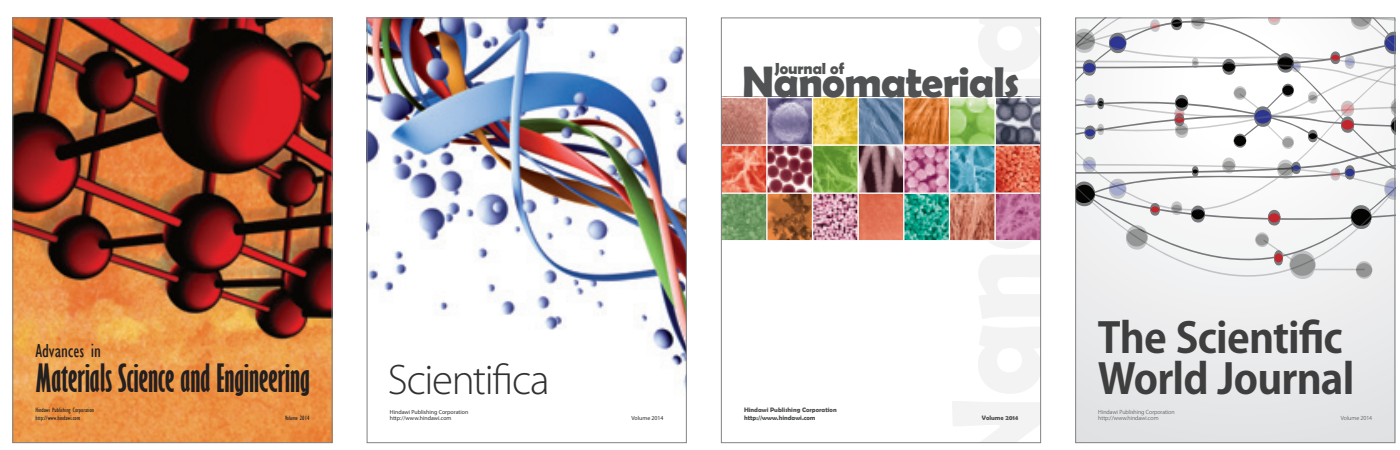

\section{The Scientific World Journal}
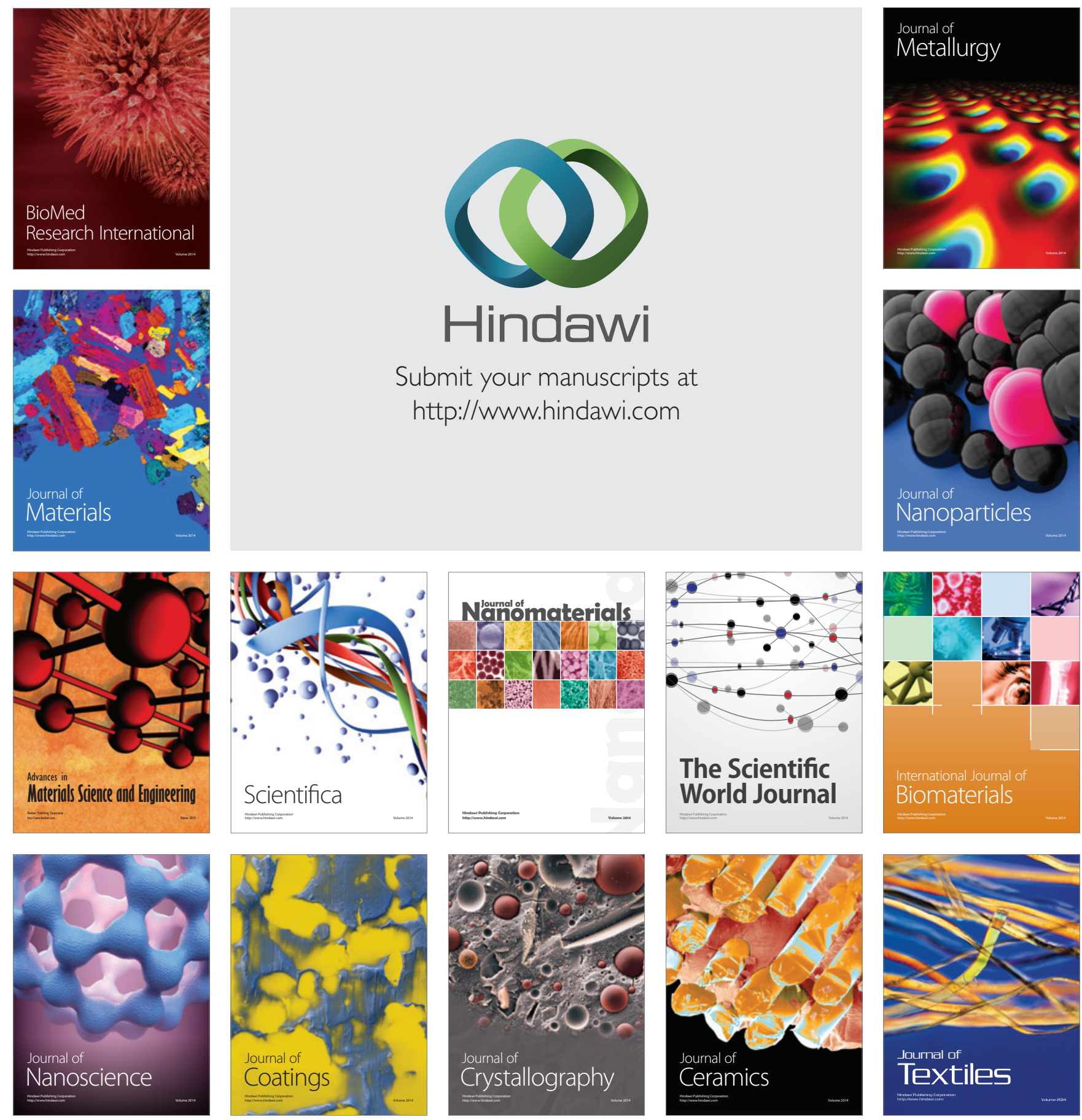